\title{
Institutional Repository
}

\section{Publication information}

\begin{tabular}{|l|l|}
\hline Title & Active control of membrane-type acoustic metamaterial by electric field \\
\hline Author(s) & Xiao, Songwen; Ma, Guancong; Li, Yong; Yang, Zhiyu; Sheng, Ping \\
\hline Source & Applied Physics Letters, v. 106, (9), March 2015, article number 091904 \\
\hline Version & Published version \\
\hline DOI & https://doi.org/10.1063/1.4913999 \\
\hline Publisher & American Institute of Physics (AIP) Publishing \\
\hline
\end{tabular}

\section{Copyright information}

This article may be downloaded for personal use only. Any other use requires prior permission of the author and AIP Publishing. The following article appeared in "Xiao, Songwen; Ma, Guancong; Li, Yong; Yang, Zhiyu; Sheng, Ping. (2015). Active control of membrane-type acoustic metamaterial by electric field, Applied physics letters, v. 106, (9), March 2015, article number 091904." and may be found at "https://doi.org/10.1063/1.4913999".

\section{Notice}

This version is available at HKUST Institutional Repository via http://hdl.handle.net/1783.1/65902

If it is the author's pre-published version, changes introduced as a result of publishing processes such as copy-editing and formatting may not be reflected in this document. For a definitive version of this work, please refer to the published version. 


\section{A P P $\mid \begin{aligned} & \text { Applied Physics } \\ & \text { Letters }\end{aligned}$}

\section{Active control of membrane-type acoustic metamaterial by electric field}

Songwen Xiao, Guancong Ma, Yong Li, Zhiyu Yang, and Ping Sheng

Citation: Applied Physics Letters 106, 091904 (2015); doi: 10.1063/1.4913999

View online: http://dx.doi.org/10.1063/1.4913999

View Table of Contents: http://scitation.aip.org/content/aip/journal/apl/106/9?ver=pdfcov

Published by the AIP Publishing

\section{Articles you may be interested in}

Acoustic superlens using membrane-based metamaterials

Appl. Phys. Lett. 106, 051901 (2015); 10.1063/1.4907634

Analytical coupled vibroacoustic modeling of membrane-type acoustic metamaterials: Plate model

J. Acoust. Soc. Am. 136, 2926 (2014); 10.1121/1.4901706

Analytical coupled vibroacoustic modeling of membrane-type acoustic metamaterials: Membrane model J. Acoust. Soc. Am. 136, 969 (2014); 10.1121/1.4892870

Active acoustic metamaterials with tunable effective mass density by gradient magnetic fields Appl. Phys. Lett. 105, 071913 (2014); 10.1063/1.4893921

Transmission loss and dynamic response of membrane-type locally resonant acoustic metamaterials J. Appl. Phys. 108, 114905 (2010); 10.1063/1.3514082

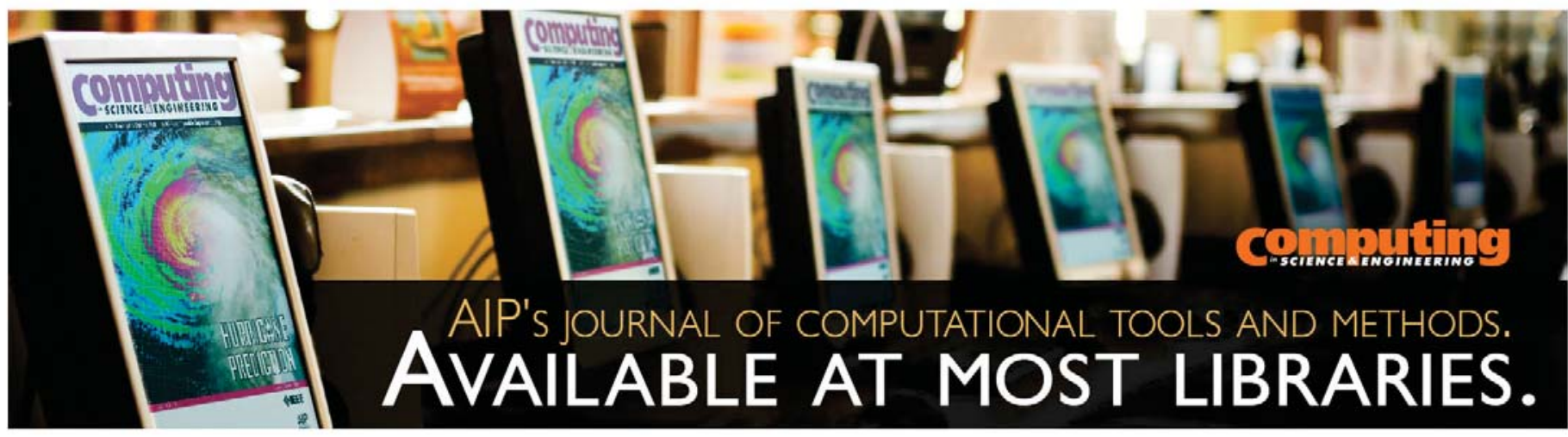




\title{
Active control of membrane-type acoustic metamaterial by electric field
}

\author{
Songwen Xiao, ${ }^{1}$ Guancong Ma, ${ }^{1}$ Yong Li,${ }^{1}$ Zhiyu Yang, ${ }^{1, a)}$ and Ping Sheng ${ }^{1,2}$ \\ ${ }^{1}$ Department of Physics, Hong Kong University of Science and Technology, Clear Water Bay, Kowloon, \\ Hong Kong, China \\ ${ }^{2}$ Institute for Advanced Study, Hong Kong University of Science and Technology, Clear Water Bay, \\ Kowloon, Hong Kong, China
}

(Received 14 August 2014; accepted 21 February 2015; published online 3 March 2015)

\begin{abstract}
By employing a metal-coated central platelet and a rigid mesh electrode which is transparent to acoustic wave, we show that the membrane-type acoustic metamaterials (MAMs) can be easily tuned by applying an external voltage. With static voltage, the MAM's eigenfrequencies and therefore the phase of the transmitted wave are tunable up to $70 \mathrm{~Hz}$. The MAM's vibration can be significantly suppressed or enhanced by using phase-matched AC voltage. Functionalities such as phase modulation and acoustic switch with on/off ratio up to $21.3 \mathrm{~dB}$ are demonstrated. (C) 2015 AIP Publishing LLC.

[http://dx.doi.org/10.1063/1.4913999]
\end{abstract}

The development of acoustic metamaterials has significantly enhanced our ability in sound wave manipulation. Their unusual constitutive effective parameters, ${ }^{1-9}$ which are not found in nature, have led to numerous remarkable phenomena, such as acoustic cloaking, ${ }^{10-14}$ acoustic focusing and imaging, ${ }^{15-18}$ nonreciprocal transmission, ${ }^{19-21}$ wave propagation and wave front engineering, ${ }^{22-24}$ and super absorption. $^{25,26}$ To date, most metamaterials are passive and hardly adjustable once fabricated. As a result, they cannot adapt to real-life scenarios that are likely to change constantly. One promising way to mitigate these problems is to incorporate active designs. ${ }^{27-32}$ Chen et al. used a gradient magnetic field to actively tune the membrane-type acoustic metamaterials (MAMs) by an external magnet. ${ }^{33}$ In this letter, we show that acoustic properties of membrane-type metamaterials can be actively controlled by external voltage to achieve a number of functionalities such as phase modulation and acoustic wave switch.

The structures consisting of decorated membrane resonators (DMRs) have been intensively studied. ${ }^{3,6,34-39}$ It is known that the low frequency transmission and reflection characteristics of a DMR are mainly determined by its first two eigenmodes. ${ }^{3}$ Transmission reaches maximum at these resonant frequencies and minimum at the so-called anti-resonance frequency between the resonant frequencies. To demonstrate the actively controllable functionality, we focus our analysis on the first eigenmode of a DMR in this work.

Our DMR comprises a circular rubber membrane with radius $R=27 \mathrm{~mm}$ and $t=0.15 \mathrm{~mm}$ in thickness. Its boundary is fixed on a solid ring and pre-stress has been applied in the membrane. A circular plastic disk with radius $r=15 \mathrm{~mm}$ and mass $m=400 \mathrm{mg}$ is attached to the center of the membrane. The surface of the disk is coated with a thin layer of gold about $20 \mathrm{~nm}$ thick by sputtering. A rigid mesh (Fig. 1(a)) also coated with gold film is placed above the disk. Large hollow area of the mesh minimizes its scattering to the passing acoustic waves. The pre-stress of the membrane varies somewhat from sample to sample by about $\pm 10 \%$ in relative

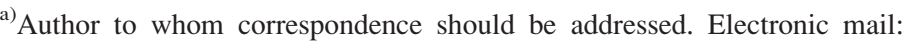
phyang@ust.hk.
}

value, which results in about $\pm 5 \mathrm{~Hz}$ shift of the first resonant frequency.

We first investigate the effect of a DC voltage $U$ across the mesh electrode and the central disk on the membrane, which serve as the two electrodes of a parallel plate capacitor. When excited by incident acoustic wave, the vibration of the membrane introduces a small harmonic variation $\Delta z$ in the distance between the electrodes. Assuming that the mesh does not deform, the electric force exerted on the disk is

$$
F_{D C} \cong-\frac{1}{2} \frac{\varepsilon S U^{2}}{(d+\Delta z)^{2}} \approx-\frac{\varepsilon S U^{2}}{2 d^{2}}\left(1-\frac{2 \Delta z}{d}\right)=-F_{0}+\bar{K} \Delta z
$$

Here, $S$ is the effective area of the disk electrode, $\varepsilon$ represents the dielectric constant of air, $U$ is the amplitude of the applied voltage, and $d$ is the separation between the mesh and the disk at zero voltage.

The electric force in Eq. (1) can be divided into two parts: a constant attractive force $F_{0}$, and a force that is linearly proportional to the disk's normal displacement $\Delta z$ with effective force constant $\bar{K}=\varepsilon S U^{2} / d^{3}$. The first term $F_{0}$ $(<0.1 \mathrm{~N}$ at $U=1000 \mathrm{~V})$ slightly shifts the equilibrium position of the membrane, whereas the second term is equivalent to an extra anti-restoring force on the disk. Since the central disk vibrates together with the membrane at the first resonance mode at $164 \mathrm{~Hz}$, it could be described by a simple springmass model with eigenfrequency $f_{1}=\frac{1}{2 \pi} \sqrt{\frac{K}{m}} \approx \frac{1}{2 \pi} \sqrt{\frac{K_{0}-\bar{K}}{m}}$, where $K_{0}$ comes from the membrane's pre-stress and could be estimated as $K_{0} \approx m\left(2 \pi f_{0}\right)^{2} \approx 425 \mathrm{~N} / \mathrm{m}$. It is then clear that the eigenfrequency will decrease as a result of the additional $\bar{K}$. On the other hand, $\bar{K}$ is inversely proportional to $d^{3}$. To maximize the effect, we choose a value $d=0.4 \mathrm{~mm}$. In that case, $\bar{K}$ is approximately $2.0 \times 10^{-4} U^{2}(\mathrm{~N} / \mathrm{m})$ with $U$ in unit of volt.

The modified impedance-tube method ${ }^{40}$ is used to obtain the transmission spectra, as shown in Fig. 1(b). The transmission peak due to the first eigenmode of the DMR is seen to red shift with increasing DC voltage. The calculated 

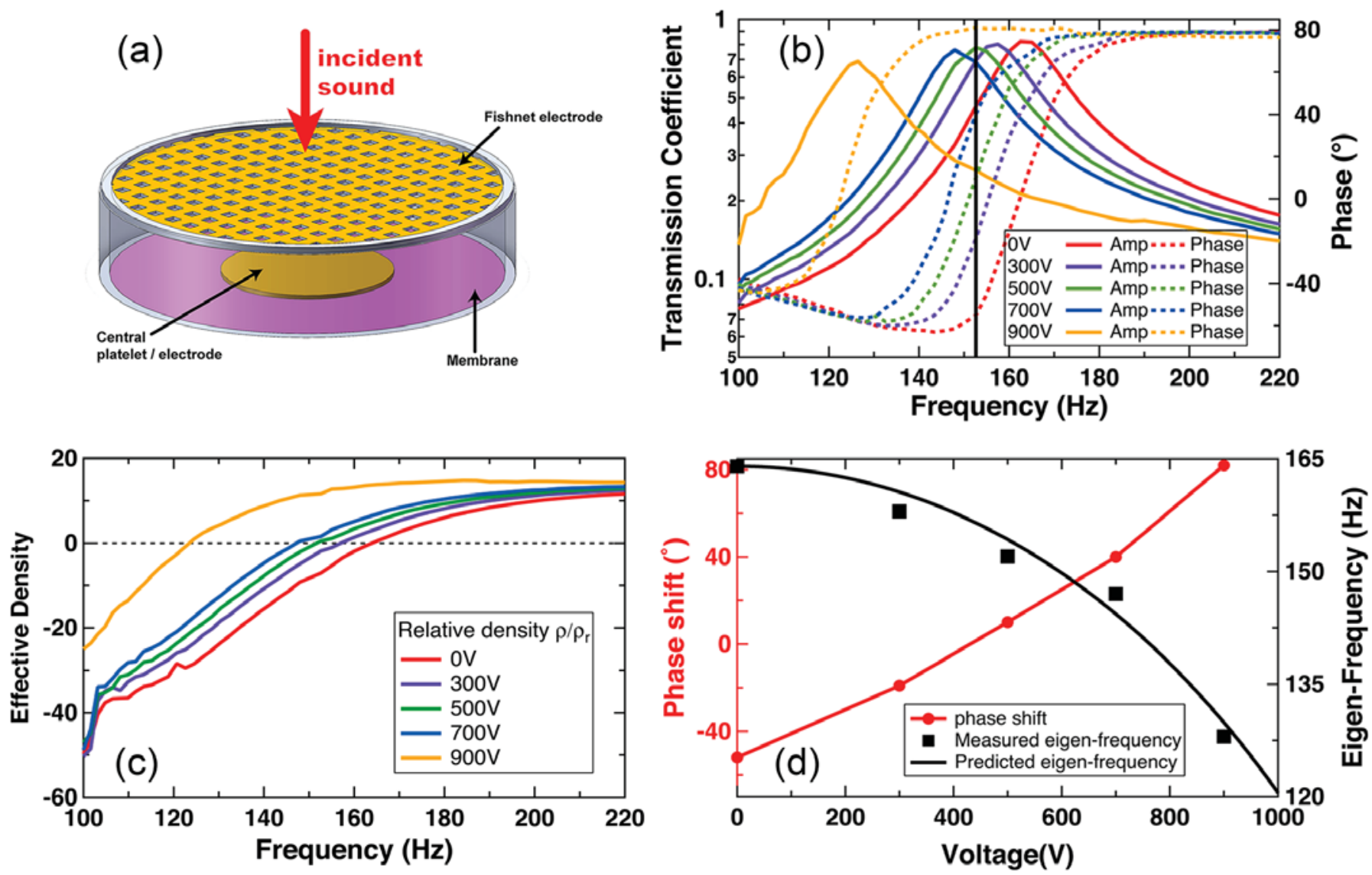

FIG. 1. (a) Schematic drawing of the sample. (b) Transmission spectra of the sample with different DC voltages. Solid curves denote the amplitude (left axis), while dashed curves (right axis) represent the phase spectra. (c) Effective mass density (relative) of the membrane system normalized to the density of rubber membrane $\left(\rho_{r}=980 \mathrm{~kg} / \mathrm{m}^{3}\right)$. (d) Phase shift (left axis) at $153 \mathrm{~Hz}$ (Black vertical line in (b)) and resonance frequency change (right axis) with voltage. The measured values are marked by black squares and the predicted resonance frequency from the spring-mass model is shown as the black curve.

effective mass density ${ }^{41}$ is shown in Fig. 1(c). It is clearly seen that the zero mass density point shifts to lower frequency with the increase of voltage. Furthermore, we show the measured eigenfrequency as a function of the DC voltage and the one predicted by the simple effective force constant $\bar{K}=\varepsilon S U^{2} / d^{3}$ in Fig. 1(d). Good agreement is obtained.

Resonant transmission of the DMR is accompanied by a $180^{\circ}$ phase change. ${ }^{3}$ With tunable eigenfrequencies, the DMR can function as an active phase modulator. As shown in Fig. 1(d), the phase of the transmitted wave can be varied continuously from $-55^{\circ}$ at zero $U$ to $81^{\circ}$ at $U=900 \mathrm{~V}$ at $153 \mathrm{~Hz}$, which is marked by the black vertical line in Fig. 1(b), a total phase shift of $136^{\circ}$. The presence of dissipation in the DMR is the reason why the phase change does not reach $180^{\circ}$.

The ability to tune the resonance frequency with static electric field allows us to construct a simple acoustic switch. Two DMRs are used, as shown in the inset of Fig. 2. The resonance frequencies of the two cells are originally set to be the same so that a single transmission peak appears at $166 \mathrm{~Hz}$. After a voltage is applied in cell 2, its resonance frequency is lowered. As stated before, ${ }^{3}$ its transmission field shall have a near $180^{\circ}$ phase change across the new resonance frequency. Hence within the frequency region between the current resonance frequencies of the two cells, the transmitted fields through these two passageways are essentially out of phase, causing destructive interference. ${ }^{26} \mathrm{~A}$ transmission dip appears at $156 \mathrm{~Hz}$ where the transmitted intensities from the two units are nearly equal. The transmission contrast over zero voltage is $21.3 \mathrm{~dB}(0.7 / 0.06)$.
We next apply AC voltage with angular frequency $\omega$ between the electrodes. The electric force on the disk can be expressed as

$$
\begin{aligned}
F_{A C} & \propto-\frac{U^{2}}{d^{2}}=-\frac{A^{2} \sin ^{2}(\omega t+\theta)}{d^{2}} \\
& =\frac{A^{2}}{2 d^{2}}[-1+\cos (2 \omega t+2 \theta)] .
\end{aligned}
$$

Here, $A$ and $\omega$ are the amplitude and the frequency of the $\mathrm{AC}$ voltage, respectively, and $\theta$ is the initial phase. Here, the effect of $\bar{K}$ is negligible because the $d=2 \mathrm{~mm}$ gap is much

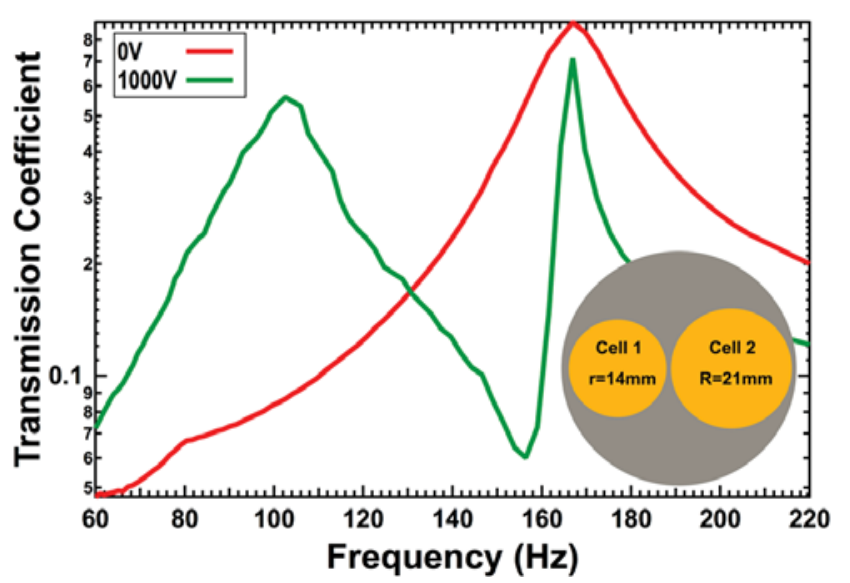

FIG. 2. Demonstration of a DC voltage controlled acoustic switch with two DMRs. The one with electrodes is cell 2, while cell 1 is passive. 
larger than that in the static case. Therefore, we could regard $d$ as a constant. The force consists of two parts: a nearly constant force and a harmonic force with angular frequency $2 \omega$. To manipulate the sound wave, this frequency $\omega$ must always satisfy the relation: $2 \omega=\omega_{s}$ where $\omega_{s}$ is the frequency of the incident sound wave.

In addition, the harmonic force is sensitive to the relative phase $2 \theta$ between the AC voltage and the incident sound wave. Its effect is seen for the first eigenmode, in which the central disk vibrates with the membrane in unison. The electric force can either enhance or suppress the vibration of the disk. By changing $2 \theta$ from 0 to $\pi$, the role of the harmonic electric force can be continuously altered from amplifier to muffler.

In order to obtain large sound transmission loss (STL), optimum amplitude of the voltage should be identified so as to totally counteract the sound pressure as well as keep the phase condition $2 \theta=\pi$. To investigate the dependence of the amplitude and the phase condition separately, we first adjust the amplitude and the initial phase of the AC voltage to obtain the highest STL of $52 \mathrm{~dB}$ as compared to zero voltage. Then, we tune the amplitude of the AC voltage while keeping the phase to its optimum value. As one can see, the STL drops quickly (red curve in Fig. 3) when the AC amplitude deviates from the optimum condition. Next, we keep the optimum amplitude of the voltage and change the initial phase (blue curve in Fig. 3). About $13 \mathrm{~dB}$ in STL is observed when the initial phase is changed by only $2^{\circ}$. This phase sensitive characteristic provides a promising method to detect small phase variations. For example, $0.025^{\circ}$ of phase shift would cause $5 \%$ relative change in transmission, which is easily detectable.

Since the vibration profile is quite similar around the resonant frequency within a wide range, the above method is applicable in the adjacent frequency region as well. Change of STL exceeding $40 \mathrm{~dB}$ can be achieved in the nearby $\pm 40 \mathrm{~Hz}$ range. Amplifying effect can also be demonstrated once we set the initial phase of the voltage so that the electric force becomes in-phase with the sound pressure.

In the example shown above, the $\mathrm{AC}$ voltage and the sound waves are generated from a single source. With the addition of an acoustic sensor and a feedback circuit, it is not

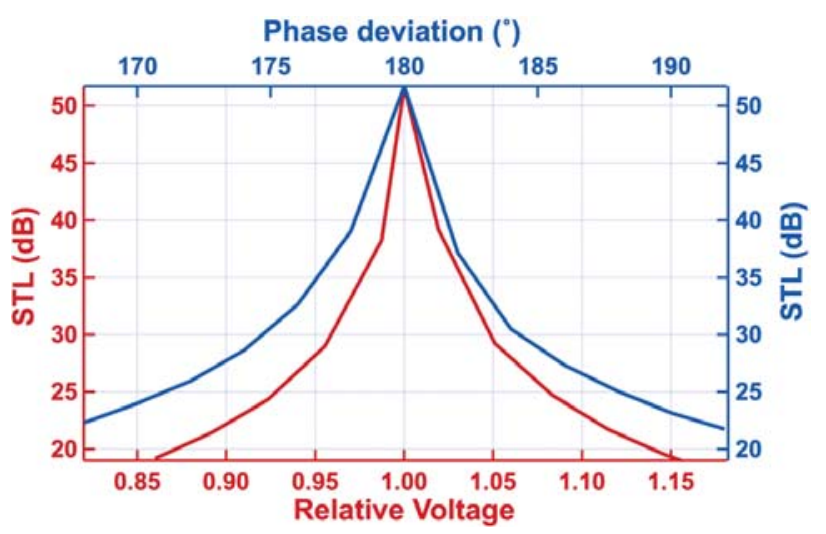

FIG. 3. The STL of the sample at the resonance frequency as compared to the transmission when no voltage is applied. The red curve is the dependence of transmission on the amplitude of AC voltage in units of the optimal voltage. The blue curve is the dependence of transmission on the initial phase. difficult to construct an active cancellation device to mute the transmission of the incident waves by generating a driving AC voltage to the DMR that is of the right amplitude and $180^{\circ}$ in phase to the incident wave. Similar devices using piezo-electric films are widely available. The advantages of our device are that it is of much larger area and that it can handle laud sound waves $(\sim 120 \mathrm{~dB})$.

Chen et al. have demonstrated a tunable resonator by using magnetic elastomer and non-uniform external magnetic field. ${ }^{33}$ The field changed the elasticity of the elastomer, and therefore the resonant features of the resonator. Although the change of field was achieved by moving a permanent magnet in this case, it is straightforward to use an electromagnet to achieve electric control of the resonant frequencies of the resonator. In our device, a force is applied directly to the resonator by external voltage, which can either tune its resonances by an additional distance-dependent DC force, or directly drive its vibration by an AC force. Our device is also more compact and light weight as compared to the magnetic resonator.

In summary, we have shown that active control of the membrane-type acoustic metamaterials can be achieved by external applied voltage. DC voltage can modulate the resonance frequency and tune the phase, serving as an active phase modulator in a phase array that could manipulate sound waves at will. AC voltage provides an extra vibration source that can act as an acoustic switch and can thereby serve as a good candidate for specific circumstances within certain frequency ranges.

Financial support for this work by HKUST 605212, HKUST2/CRF/11G, and AoE/P-02/12 from the Hong Kong SAR Government is gratefully acknowledged.

${ }^{1}$ Z. Liu, X. Zhang, Y. Mao, Y. Y. Zhu, Z. Yang, C. T. Chan, and P. Sheng, Science 289, 1734 (2000).

${ }^{2}$ N. Fang, D. Xi, J. Xu, M. Ambati, W. Srituravanich, C. Sun, and X. Zhang, Nat. Mater. 5, 452 (2006).

${ }^{3}$ Z. Yang, J. Mei, M. Yang, N. Chan, and P. Sheng, Phys. Rev. Lett. 101, 204301 (2008).

${ }^{4}$ S. H. Lee, C. M. Park, Y. M. Seo, Z. G. Wang, and C. K. Kim, Phys. Rev. Lett. 104, 054301 (2010).

${ }^{5}$ Y. Lai, Y. Wu, P. Sheng, and Z. Q. Zhang, Nat. Mater. 10, 620 (2011).

${ }^{6}$ M. Yang, G. Ma, Z. Yang, and P. Sheng, Phys. Rev. Lett. 110, 134301 (2013)

${ }^{7}$ Z. Liang and J. Li, Phys. Rev. Lett. 108, 114301 (2012).

${ }^{8}$ Z. Liang, T. Feng, S. Lok, F. Liu, K. B. Ng, C. H. Chan, J. Wang, S. Han, S. Lee, and J. Li, Sci. Rep. 3, 1614 (2013).

${ }^{9}$ Y. Xie, B. I. Popa, L. Zigoneanu, and S. A. Cummer, Phys. Rev. Lett. 110, 175501 (2013).

${ }^{10}$ H. Chen and C. T. Chan, J. Phys. D: Appl. Phys. 43, 113001 (2010).

${ }^{11}$ S. Zhang, C. Xia, and N. Fang, Phys. Rev. Lett. 106, 024301 (2011).

${ }^{12}$ B. I. Popa, L. Zigoneanu, and S. A. Cummer, Phys. Rev. Lett. 106, 253901 (2011).

${ }^{13}$ L. Sanchis, V. M. García-Chocano, R. Llopis-Pontiveros, A. Climente, J. Martínez-Pastor, F. Cervera, and J. Sánchez-Dehesa, Phys. Rev. Lett. 110, 124301 (2013).

${ }^{14}$ L. Zigoneanu, B. I. Popa, and S. A. Cummer, Nat. Mater. 13, 352 (2014).

${ }^{15} \mathrm{~J}$. Li, L. Fok, X. Yin, G. Bartal, and X. Zhang, Nat. Mater. 8, 931 (2009).

${ }^{16}$ S. Zhang, L. Yin, and N. Fang, Phys. Rev. Lett. 102, 194301 (2009).

${ }^{17}$ J. Zhu, J. Christensen, J. Jung, L. Martin-Moreno, X. Yin, L. Fok, X. Zhang, and F. J. Garcia-Vidal, Nat. Phys. 7, 52 (2011).

${ }^{18}$ F. Lemoult, M. Fink, and G. Lerosey, Phys. Rev. Lett. 107, 064301 (2011).

${ }^{19}$ B. Liang, B. Yuan, and J. C. Cheng, Phys. Rev. Lett. 103, 104301 (2009). 
${ }^{20}$ B. Liang, X. S. Guo, J. Tu, D. Zhang, and J. C. Cheng, Nat. Mater. 9, 989 (2010).

${ }^{21}$ X. F. Li, X. Ni, L. Feng, M. H. Lu, C. He, and Y. F. Chen, Phys. Rev. Lett. 106, 084301 (2011)

${ }^{22}$ Y. Li, B. Liang, Z. M. Gu, X. Y. Zou, and J. C. Cheng, Sci. Rep. 3, 2546 (2013).

${ }^{23}$ P. Zhang, T. Li, J. Zhu, X. Zhu, S. Yang, Y. Wang, X. Yin, and X. Zhang, Nat. Commun. 5, 4316 (2014)

${ }^{24}$ F. Lemoult, N. Kaina, M. Fink, and G. Lerosey, Nat. Phys. 9, 55 (2013).

${ }^{25}$ J. Mei, G. Ma, M. Yang, Z. Yang, W. Wen, and P. Sheng, Nat. Commun. 3, 756 (2012)

${ }^{26}$ G. Ma, M. Yang, S. Xiao, Z. Yang, and P. Sheng, Nat. Mater. 13, 873 (2014).

${ }^{27}$ A. A. Govyadinov, V. A. Podolskiy, and M. A. Noginov, Appl. Phys. Lett. 91, 191103 (2007).

${ }^{28}$ Z. Wang, Y. Luo, L. Peng, J. Huangfu, T. Jiang, D. Wang, H. Chen, and L. Ran, Appl. Phys. Lett. 94, 134102 (2009).

${ }^{29}$ A. R. Katko, S. Gu, J. P. Barrett, B. I. Popa, G. Shvets, and S. A. Cummer, Phys. Rev. Lett. 105, 123905 (2010).

${ }^{30}$ B. I. Popa, L. Zigoneanu, and S. A. Cummer, Phys. Rev. B 88, 024303 (2013).

${ }^{31}$ B. I. Popa and S. A. Cummer, Nat. Commun. 5, 3398 (2014).
${ }^{32}$ Q. Ma, Z. L. Mei, S. K. Zhu, T. Y. Jin, and T. J. Cui, Phys. Rev. Lett. 111, 173901 (2013)

${ }^{33}$ X. Chen, X. C. Xu, S. G. Ai, H. S. Chen, Y. M. Pei, and X. M. Zhou, Appl. Phys. Lett. 105, 071913 (2014).

${ }^{34}$ G. Ma, M. Yang, Z. Yang, and P. Sheng, Appl. Phys. Lett. 103, 011903 (2013).

${ }^{35}$ Z. Yang, H. Dai, N. Chan, G. Ma, and P. Sheng, Appl. Phys. Lett. 96, 041906 (2010)

${ }^{36}$ J. Mei, G. Ma, M. Yang, J. Yang, and P. Sheng, in Acoustic Metamaterials and Phononic Crystals, edited by P. A. Deymier (Springer, Berlin, Heidelberg, 2013).

${ }^{37}$ C. J. Naify, C. M. Chang, G. McKnight, F. Scheulen, and S. Nutt, J. Appl. Phys. 109, 104902 (2011).

${ }^{38}$ C. J. Naify, C. M. Chang, G. McKnight, and S. Nutt, J. Appl. Phys. 110, $124903(2011)$

${ }^{39}$ C. J. Naify, C. M. Chang, G. McKnight, and S. Nutt, J. Appl. Phys. 108, $114905(2010)$

${ }^{40}$ K. M. Ho, Z. Yang, X. X. Zhang, and P. Sheng, Appl. Acoust. 66, 751 (2005).

${ }^{41}$ V. Fokin, M. Ambati, C. Sun, and X. Zhang, Phys. Rev. B 76, 144302 (2007). 